\title{
Miranda
}

Revue pluridisciplinaire du monde anglophone /

Multidisciplinary peer-reviewed journal on the English-

speaking world

$20 \mid 2020$

Staging American Nights

\section{La nuit de la langue. Intimité poétique de Fabio Morábito}

\section{Nathalie Galland}

\section{OpenEdition}

\section{Journals}

Édition électronique

URL : http://journals.openedition.org/miranda/25709

DOI : 10.4000/miranda.25709

ISSN : 2108-6559

\section{Éditeur}

Université Toulouse - Jean Jaurès

\section{Référence électronique}

Nathalie Galland, "La nuit de la langue. Intimité poétique de Fabio Morábito », Miranda [En ligne], 20 | 2020, mis en ligne le 24 mars 2020, consulté le 16 février 2021. URL : http://journals.openedition.org/ miranda/25709; DOI : https://doi.org/10.4000/miranda.25709

Ce document a été généré automatiquement le 16 février 2021.

\section{(c) (i) (5)}

Miranda is licensed under a Creative Commons Attribution-NonCommercial-NoDerivatives 4.0 International License. 


\title{
La nuit de la langue. Intimité poétique de Fabio Morábito
}

\author{
Nathalie Galland
}

"C'est à partir de phrases très simples dans leur raccourci - et qui sont des paliers de pénombre - ; que mes livres ont été édifiés. Ainsi, j'ai appris que la nuit est le lieu de l'écrivain ». Ces mots d'Edmond Jabès ${ }^{1}$ révèlent le chemin d'écriture que partage, entre autres flâneurs noctambules, Fabio Morábito. L'imaginaire métapoétique de l'écrivain italo-mexicain compose un paysage profondé par la nuit, dans des touches, des figures dispersées au gré des poèmes, récits, essais et autres nouvelles ${ }^{2}$.

Arrière-pays aux nocturnités ${ }^{3}$ multiples, la nuit accompagne ou déclenche presque toujours la venue d'une écriture faite de matière noire: "Hay en todo artista un marginado de la tribu y hay en todo arte, por su mixtura de realidad y ensueño, un halo de penumbra al mediodía $»^{4}$. Comme geste syncrétique, l'écriture abolit bien les catégories hermétiques du diurne et du nocturne en imposant l'ombreux dans le séjour de la lumière. Soustraits à la temporalité par la contagion de l'obscur, le geste d'écrire et la page même « rendent la nuit présente $»^{5}$.

Nous parlerons ainsi d'abord de nuit de l'écriture: temporalité du geste et matière d'encre des vers diront le régime nocturne de la création poétique. En écrivant avant le jour, le poète est à la nuit, éclipsé et libre de tâtonner, de s'acheminer. Mais s'imposera aussi une nuit de la langue, car il est chez Morábito une condition cosmopolite qui brouille le rapport au langage. Né à Alexandrie d'une famille italienne - son enfance sera effectivement milanaise - Fabio Morábito écrit dans l'espagnol du Mexique où il est arrivé à 15 ans. Depuis Lotes baldíos (1985) le premier des quatre recueils publiés ${ }^{6}$, l'écriture poétique se pense et se construit comme un entre-deux langues, écriture aux confins du premier idiome, l'italien familial et nomade, déjà baigné de lointain, et d'un espagnol malgré lui palimpseste du souffle intime. Le geste lyrique aussi semble interroger toujours, dans l'élan des vers, la part de l'avancée et celle du retournement.

Lève-tôt plutôt que couche-tard, Morábito sera ici d'abord un veilleur aux confins ${ }^{7}$, à la fois veilleur et éveilleur de la sensibilité nocturne, éveilleur de la langue en contre-jour du poème et voyeur d'avant le jour. Osant la nuit comme expérience libertaire, " 
balbuceo liberador $\|^{8}$, et promesse de création, le poète se fera tout entier être nocturne, poète vagabond jouant de l'obscur, voleur furtif de mots et d'images ou vampire iconique.

Il sera enfin un "effaçonneur ", faisant de la nuit de la langue, de la nocturnité de l'italien laissé derrière, la respiration profonde, la rythmique secrète et perturbante de poèmes outre-noirs, de textes d'outre-nuit au destin nomade.

\section{(É)veilleur}

Salí a caminar a las 5:40 am. [...] Lo hice tanto en invierno, mucho antes de que saliera el sol, a ocho o diez grados bajo cero [...]. Me producía un intenso placer caminar en medio de ese silencio, mientras la inmensa mayoría de la gente seguía metida en su cama. Ahora veo que esas caminatas eran una forma de despertar a fondo, o sea de empezar a escribir, de calentar la pluma. ${ }^{10}$

Ce sont quelques lignes tirées de También Berlín se olvida (México : Sexto Piso, 2006), une somme de brefs récits où Fabio Morábito mêle intimement expérience de l'ailleurs et geste de l'écriture. "Retranch[é] du peuple des endormis " ${ }^{11}$, le poète marche dans Berlin. Dans l'anonymat silencieux d'avant le jour, tout contre la vie ordinaire et momentanément exposé à l'intempérie, il goûte une solitude inaugurale où précisément il gagne comme différence son identité d'écrivain. La langue peut s'originer à nouveau dans l'exercice. Se tissent des correspondances (naturelles, corporelles) entre rythme des pas, battements intimes et la caisse de résonance linguistique que constituent ensemble le corps et la nuit: mise en marche du matériau informe et latent qui deviendra poème, quelque chose qui se chauffe avant un possible déploiement, inflammation poétique. Ces liens entre marcher et écrire saisis par une promenade nocturne «à la fois synesthésique et cinétique $»^{12}$, rappellent les notes de Jacques Roubaud ou de Jean-Pascal Dubost sur le même thème :

Marcher, pour solliciter les pensées, dont on sait la présence, mais lointaine, les soulever du fond du corps [(« despertar a fondo »)]; [...] le poème est une présence vague et floue, informe, par conséquent insatisfaisante, mais sue; aller pour trouver une pleine disposition de ses moyens; la marche récupère le corps, l'éveille : cet état-ci est rêverie : éveil concentré, le même qui opère lors de l'acte d'écrire. Le corps se récupère, et récupère en lui le matériau indistinct. ${ }^{13}$

Premier territoire du langage, corps, palpitation, respiration : ce n'est pas un hasard si l'éveil du corps est éveil de la langue, "cuestión de pulso ", "sangre de la propia sangre $»^{14}$, inicium et initiation, résonance d'un lyrisme du sang.

Pour autant, il est aussi chez Fabio Morábito une discipline de l'obscur, une « adhésion obstinée à la nuit ${ }^{15}$. S'il marche comme les autres dorment, c'est pourtant dans l'intimité de l'appartement que se construit le plus souvent cette écriture a deshoras, contre le jour, en contre temps, enjeu de l'envers profond :

Puesto que escribo en una lengua

que aprendí,

tengo que despertar

cuando los otros duermen.

Escribo como quien recoge agua

de los muros,

me inspira el primer sol

de las paredes.

Despierto antes que todos,

pero en alto. 


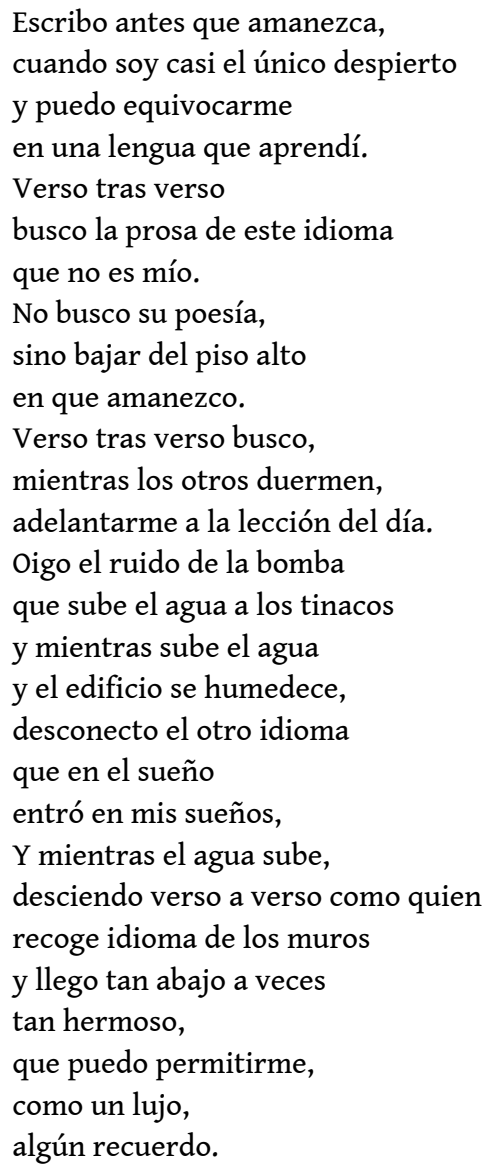

Engagé dans une collecte sensible du nocturne, souvent à l'orée du visible et de l'audible et rendue dans des collections d'images et de sons (lumière projetée de la lampe, bruits de tuyauterie, rumeurs sourdes de la ville), le poète glisse à une collecte de l'invisible, qui redit l'emmêlement intime des langues.

C'est bien la condition du bilinguisme qui pousse à la nocturnité du geste d'écrire, véritable ressort de l'écart, jeu de graphème du clair et du sombre (« el primer sol de las paredes »/«la lección del día»). Veiller à l'heure où dort encore le reste du monde, c'est interpréter sa différence en la liant tout entière à la langue : la nuit de l'écriture dit le temps singulier, temps du poème, temps du poète, autre du temps des autres, contre-jour où s'aveugle la langue première, contre temps où peut librement affleurer le litige poétique. En habitant ces heures plus lentes où la langue d'adoption est vouée à la nuit, la menace de l'équivoque s'abolit en effet momentanément. À cette série de " contre» nocturnes, il faudrait ajouter aussi le contrepoids des langues et des imaginaires. Le poème cherche à s'acheminer, s'avancer vers le jour de la langue laissant l'italien redescendre en sa nocturnité, regagner les lointains profonds de l'origine. Dans la composition poétique, le bougé des langues et celui du monde, semblent se faire à la poulie, par versements réciproques : contrepoids, dialogue " lengua/agua ", parcours croisés de l'eau qui monte et des mots qui descendent en quête $\mathrm{du}$ poème, de la trivialité où batailler plus tard avec le jour («y mientras sube el agua / y el edificio se humedece, / desconecto el otro idioma »). Quête du souffle prosaïque de la langue d'usage (qui est aussi la langue du poème), incertain véhicule, et double oublieux de la langue de l'origine, quête pour tracer le parcours de ce qui chancelle, vers plus de solidité : «[...] desciendo verso a verso como quien recoge idioma de los muros / y llego tan abajo a veces, tan hermoso, / que puedo permitirme, /como un lujo 
/algún recuerdo ». Cet acheminement nocturne, de vers en vers figurant à leur tour des "paliers de pénombre», manifeste aussi l'intense capillarité des mots, leur subtile liquidité, leur qualité d'écoute. «Se escribe poesía a pesar de la escritura, a contrapelo de la sordera de la escritura, en contra de la arritmia y de la techumbre de la escritura $»^{16}$ dit ailleurs Morábito, renouant avec la friction des contraires, et inscrivant l'élan poétique comme retournement précaire dans la profondeur.

Comme toile tremblante, espace de domestication de la précarité du dire poétique, la nuit traverse le poème au gré de boiteries qui configureront à l'échelle de l'œuvre une poétique de la labilité :

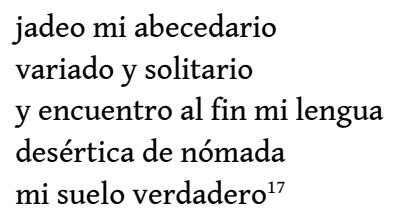

Quête du repos et de la stabilité, mais sur la faille pourtant - frictions intimes et secousses sismiques de l'Anáhuac - le poème lui-même est une traversée : il gagne en tension, questionne ses confins, entre immédiateté et étrangeté, proche et lointain. Dans la nuit de Mexico, montent ainsi des exhalaisons d'obscurités anciennes : celles de deux autres villes, paysages tutoyés et enchâssés d'une cartographie littéraire intime et intensément mobile. La langue du poème, langue-rhapsodie qui génère à la fois raisonnement et résonance, se fait dès lors substrat de mondes lentement adjointés :

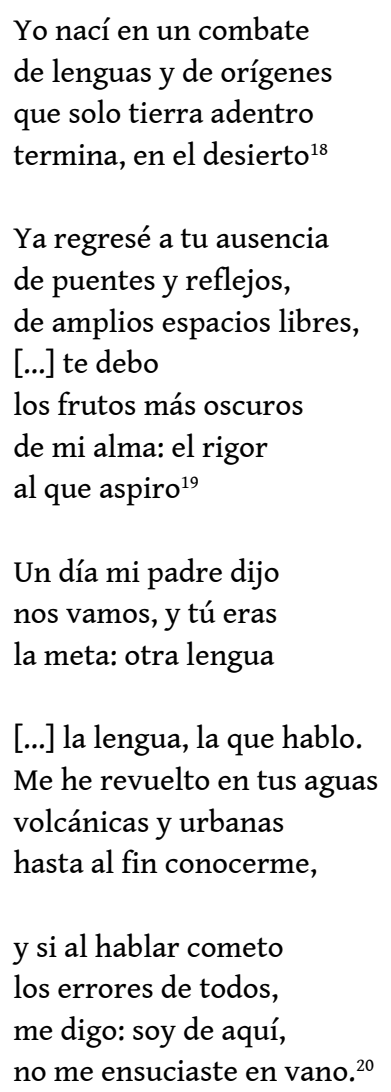

C'est un triptyque articulé par la langue et l'identité nomade: Alexandrie, ou la cosmogonie intime d'un être au monde d'emblée prisonnier du multiple, que seul le désert est capable d'assécher; Milan, ville d'absences miroitantes et complexes, labyrinthe qui pousse à la profondeur et la contention ${ }^{21}$; Mexico, ville volcan en contagion, impur espace de saisie de soi. Ces exils successifs, pensés a posteriori comme 
voyage vers la langue, errance du poète vers une vague terre promise depuis l'univers syncopé des signes (" me he revuelto", " cometo/los errores de todos»), rappellent la tension même du geste d'écrire, où l'on entendra Henri Meschonnic :

Écrire ne se fait pas dans la langue, comme si elle était maternelle, donnée, mais vers la langue. Écrire n'est peut-être qu'accéder, en s'inventant, à la langue maternelle. Écrire est, à son tour, maternel, pour la langue. ${ }^{22}$

La nuit, lieu de l'écart, acheminement. L'écriture là est libre de saisir ce qui surgit depuis l'intime altérité: matières et temps logés dans le signe, résonance du nomadisme. Elle favoriserait presque dans l'invisible vis-à-vis des langues, le juste visà-vis du monde si précisément, contaminante dans sa noirceur, elle ne transformait le veilleur en voyeur.

\title{
Du veilleur au voyeur
}

\author{
Ventanas encendidas, mi tormento. \\ Gente sólo visible en esta hora. \\ De día los edificios son triviales, \\ de noche la fragilidad de su interior me hechiza. \\ Se espía buscando desnudeces, \\ pero también por hambre de poesía, \\ hambre no de la piel del otro, \\ sino de una manera de gastar latidos, \\ de ver cómo transcurre un corazón ajeno. \\ Por eso morbo y poesía andan juntos. \\ Falta de prosa, mi tormento. ${ }^{23}$
}

Trois fenêtres, la nuit. Nights windows (1928), le tableau bien connu d'Edward Hopper sert aussi d'illustration de couverture à la deuxième anthologie ${ }^{24}$ de Fabio Morábito, Ventanas encendidas, parue en 2012 aux éditions Visor de Poesía (Madrid). Appartenant à l'ombre, le poète observe, abolit quelques frontières, excite. Les fenêtres éclairées, comme "puissance fictionnelle d'apparitions $»^{25}$, trouvent dans le poème leur reflet d'encre. Le ressort du texte est en effet essentiellement visuel, qui construit une érotique à la longue vue, révélant le plaisir assumé, grâce à la nuit, de qui transgresse l'intimité, et en aval le plaisir du texte excité par le besoin de rythme ("gastar latidos " assez masturbatoire). Ce sortilège nocturne du monde en métamorphose est un détonateur poétique, une manière de creuser la nuit dans la fenestritée ${ }^{26} \mathrm{du}$ texte. Aux heures arrachées au jour, aux apparitions qu'elles permettent, répond un autre comput poétique, ultime réseau du noir avant l'aube, temps nocturne in extremis : plus qu'écart alors, la nuit est ouvroir au poème. Comme le regard, les vers avancent dans le noir, se risquent ailleurs :

[...] el poeta sólo sabe, de lo que escribe, el verso que lo tiene ocupado, y más allá de él no sabe nada: así, cada nuevo verso lo toma de sorpresa. [...] La poesía es como un hombre en una cueva oscura, que antes de dar el siguiente paso debe afianzar ambos pies y encomendarse a $\operatorname{Dios}^{27}$.

Entre surprise de l'incertain, terra incógnita du vers à venir saisi comme à tâtons, et risque de l'abîme, menace du temps nocturne et poussée exophorique qui invite à la transfiguration, la figure du poète se change justement en étrange oiseau de nuit :

[...] porque cuando se escribe con intensidad se está robando, sustrayendo de los bolsillos del lenguaje las palabras necesarias para aquello que uno quiere decir, justo esas palabras y ni una más. Todavía hoy, después de muchos años, acostumbro levantarme muy temprano para escribir, cuando todo el mundo está dormido. No 
concibo la escritura como una actividad preclara, sino furtiva.

[...] A fuerza de vigilarse mutuamente, centinelas y ladrones han terminado por parecerse y de lejos es difícil saber quién es quién. El escritor, en cierto modo, los fusiona, porque protege y roba, sustrae y aprovisiona al mismo tiempo. Escribo cuando los demás duermen todavía y por lo tanto escribo para que nadie despierte, para que sigan dormidos. Soy el que protege pero también el que acecha, el que le cuida la espalda a los otros y el que escribe a sus espaldas, la cabeza inclinada sobre la escritura, como sólo la escritura es capaz de inclinar una cabeza. ${ }^{28}$

Poète voleur, d'abord, recéleur de mots, affairé et furtif. Détrousseur et sentinelle ensuite, dans une emblématique condition hybride. Renouant en effet avec la figure du brigand $^{29}$, double maléfique du poète innocent qui cherche la nuit ce qu'il ne peut trouver le jour, Morábito établit différentes connexions entre écriture et imposture. À partir de formes désacralisantes (matérialistes, instrumentales) des représentations du geste lyrique et du poète en hors-la-loi du jour, il impose une «beauté du trivial ${ }^{30}$, une manière d'habiter la nuit dans laquelle le litige est encore au cœur de l'acte d'écrire.

D'autres fois, de cette galerie de portraits, se soulève un poète au sang d'encre :

Pero quien nace dos veces carece de acento nativo y lo que busca Drácula es eso, el acento local, el secreto del habla. El escritor afincado en otro idioma busca lo mismo y comparte la misma palidez lingüística, que en él suele traducirse en un exceso de estilo, o sea un exceso de máscara, para ocultar, como el vampiro, su condición de parásito. ${ }^{31}$

Avec cette transfiguration nocturne du poète en vampire iconique, il ne s'agit plus simplement de faire varier les manières d'habiter la nuit qui sont autant de manières de considérer le geste d'écrire, mais de rendre compte, par la métaphore, de la condition précaire du nomadisme linguistique. En usant d'une langue-sangsue, langue d'adoption buvant de la prose environnante, de «l'accent local », du « secret » de ce qui est maternel aux autres, le poète tâche de se fondre, de s'enfoncer dans le jour depuis l'obscur.

S'il est tour à tour voyeur et jouisseur tout à son aise dans les replis nocturnes, peu regardants des codes, et qui s'amuse d'avoir " mauvais genre $»^{32}$, le poète habite la nuit d'abord parce que ses déclinaisons de nocturnité font sens à son expérience cosmopolite, à la vulnérabilité ressentie d'une écriture à double fond mémoriel. Désir de nuit et loi de la nuit agissent ainsi dans le poème comme acheminement, traversée, ouvroir des ressorts intimes, affleurement du souffle, questionnement de l'élan et de la part nocturnes de la langue.

\section{Effaçonneur}

Il faudrait repartir de l'idée que la langue se souvient, que l'espagnol du Mexique, qui est la langue exclusive de l'écriture pour Morábito, contient un peu de nuit, que le poème est bien le résultat du travail nocturne. "Terre d'outre-nuit " pour Jabès ${ }^{33}$, produit d'une lente assimilation pour Morábito :

COMO DELANTE de un prado una vaca

que inclina mansamente la cabeza

y solo la levanta para contemplar su suerte,

o una ballena estacionada justo

en la corriente de una migración de plancton,

a veces me sorprendo estático y hundido,

estacionado en medio del gran prado del lenguaje 
$[\ldots]$

Por eso escribo: para recobrar

del fondo todo lo adherido,

porque es el único rodeo en el que creo,

porque escribir abre un segundo estómago

en la especie.

El verso con su ácido remueve las partículas

dejadas por el plancton de los días

y a mí también, como el cetáceo,

me sale un chorro a veces,

una palabra vertical que rompe el tedio de los mares. ${ }^{34}$

Gigantesque vache marine broutant des mots et du souffle, la baleine pour Morábito devient nouveau miroir de l'écriture, dérivant geste lyrique et son processus de filtration, de sélection patiente et obstinée, d'expression-expulsion. Et ce geste poétique s'épanouit comme effaçonnement. On doit le terme à Jean Portante, Luxembourgeois d'origine italienne qui écrit en français (et est aussi traducteur de l'espagnol), et qui porte en lui un désa/encrage, un décentrement comparable à celui de Morábito :

comme la baleine, mon écriture cachait en elle, alors que d'aspect elle était française, le poumon d'une autre langue. Que mon écriture était de forme française, mais de respiration autre dont la matérialité du texte ne savait être qu'une traduction plus ou moins efficace. Et dont le processus devait être dissout - comme l'aspirine - si je ne voulais pas succomber à la schizophrénie.

La baleine [...] incarnant le partant qui n'arrive jamais, devenait soudain mon instrument principal pour lire en moi le travail de l'écriture. Elle m'a permis de forger un verbe nouveau, devenu le titre d'un de mes livres: Effaçonner. Dans le sacrifice on donne et on prend. C'est ce qu'a fait la baleine. Elle a donné et pris. Elle s'est effaçonnée. Elle a effacé une forme pour s'en façonner une autre. Mais, tout en faisant cela, elle a gardé l'essentiel de son état initial, le poumon ${ }^{35}$.

Entre effacement et façonnage, un « chorro a veces,/ una palabra vertical que rompe el tedio de los mares", s'élabore la langue de Fabio Morábito, comme nuit intime qui migre, transhume au cœur des mots, et devient une langue-baleine qui «poumonne». Le vers, la voix, le souffle se disent comme "inhalation profonde", "exhalaison mélancolique $\aleph^{36}$, invention de silence, immersion dans la profondeur ${ }^{37}$. L'effaçonner, comme nuit de la langue, processus de création poétique, dit ainsi l'âpre emmêlement interculturel, le cheminement d'une voix mouvante pour se dire et se traduire en quelqu'un d'autre. Langue baleine, longue haleine du temps qui s'alimente du monde par scrupuleuse sélection, ample navigation qui puise entre deux eaux, qui s'étrangéise dans la persistance du rythme, le poète l'évoque dans la récurrence de la profondeur :

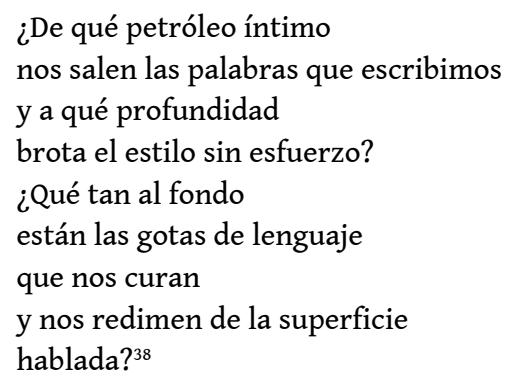

Avec la viscosité de ce pétrole intime, c'est la matière ombreuse, la descente dans la profondeur originelle qui se dit, où atteindre nouvellement énergie, combustible à la langue, magma vibrant: «los versos de la poesía, que se resisten a convertirse en renglones, alientan nuestra respiración perdida $»^{39}$. Redonner du souffle au rythme 
perdu, atteindre l'abîme où demeure la respiration naturelle, diastole systole ou inspiration expiration, là où la langue en effet "poumonne ». La nuit de la langue dira ainsi la résonance intime perçue par le poète guetteur, le bruissement de ce qui est absent désormais, mais fait toujours présence à l'oreille.

Puisque «l'oreille est le sens de la nuit ${ }^{40}$, on pensera aux principes de l'acousmatique définis, notamment, par Patrick Quillier. Acousmatique, ce " for intérieur résonnant »" ce qui est amplifié par l'obscurité et circule dans l'entre, rythme turbulent où mots, images, sons entrent en résonance. Bruissement du passage au centre de la création poétique de Morábito. Comme manifestations dans le poème de ce for intérieur par définition intime, et donc en partie indirect et caché, on saisira ici le rythme de vers qui interrogent, comme harcelés par l'incertain. Une tension également augmentée par l'enjambement quand les vers ne se soumettent pas à la clôture de la ligne, à l'immédiat. Et puis il y a, comme une constellation de figures et de paysages marins, la circulation intertextuelle des symboles de l'écriture: mots-pétrole, profondeur mémorielle, nostalgie des rives - Alexandrie peut-être -, la langue comme guérison des fissures intimes.

Effaçonnement alors le poème, tiré de l'expérience nomade, né d'un toucher du monde multiple, les villes, l'ailleurs emporté avec soi. Alexandrie, Milan, Mexico, et avec ces villes, bribes de continents, la petite enfance, l'enfance, l'adolescence, les présences sonores, alphabets et imaginaires multiples. Sur cette marge ombreuse et instable du bilinguisme, s'ouvre un espace de dialogue et de collision, d'altération, mais aussi de contagion, un entre où circule justement le corps sonore de la poésie.

la franja dudosa a la que me ha relegado mi bilingüismo. En ella se reúnen y dialogan dos idiomas mermados: el materno, por hallarse en continuo proceso de erosión, y el adquirido, porque no logrará jamás hacer desaparecer el fantasma del otro. $^{42}$

Entre érosion du premier alphabet et obsession du mot juste : une marge d'expérience équilibriste dans la langue. Il est saisissant de constater combien cette trajectoire à la fois existentielle et langagière - d'extranjero, expatriado, extraviado, extraterritorializado transpire dans la production littéraire de Fabio Morábito. D'abord dans la propension du texte (nouvelle, roman, essai) à devenir ou à incorporer récits de voyage, chroniques de l'ailleurs où bataillent obscurément langues et origines, des mots inapaisés. Territoire premier cratérisé, bousculé, pour évoquer l'imaginaire pierreux, perforé, explosif de César Vallejo ${ }^{43}$, absolument instable. On se souviendra justement de l'instabilité irréparable racontée par Claude Esteban dans Le Partage des mots :

\begin{abstract}
Encore faut-il que le langage où l'enfant s'aventure constitue à ses yeux une manière de totalité bienveillante, un lieu unique, irrécusable, que le doute n'habite pas ni le péril des équivoques. À chaque chose, l'exacte répartie des mots; à chaque mot, une place dans l'immense vocabulaire du monde. Un tel bonheur ne m'est point échu. Dès les premiers moments de mon expérience balbutiante, il m'a fallu chercher un chemin à travers deux idiomes qui s'affrontaient dans mon esprit, m'imposant leurs directives divergentes, leurs codes et leurs déchiffrements singuliers ${ }^{44}$.
\end{abstract}

Le parcours poétique de Fabio Morábito semble emprunter des sentiers d'une contrebande nocturne comparable, entre une italianité spectrale (territoire des morts et des mystères, obscurités des expériences et des mots anciens), rendue « raquítica y dudosa $»^{45}$ par le lointain des jours :

Así, si tú te vas,

idioma de mi lengua, 
razón profunda

de mis torpezas

y mis hallazgos,

¿con qué me quedo?

¿con qué palabras

recordaré mi infancia,

con qué reconstruiré

el camino y sus enigmas?

¿Cómo completaré mi edad? ${ }^{46}$

et une mexicanité du présent, dont la langue demeure étrange, étrangère, «lengua torpe », «mi lengua impura $»^{47}$, langue entravée par la menace du gouffre, le prisme permanent de l'erreur. "En todo hay un abajo,/un atrás de, un fondo $»^{48}$ disent deux vers, rappelant cette présence d'un ailleurs de la langue dans la langue, à la fois errance, éclat et profondeur. Et c'est cette précarité intime « qui ébranle les assises de sa présence au monde $»^{49}$.

Entre le proche et le lointain, l'ailleurs toujours renouvelé dans l'horizon des villes, l'écriture capte justement des fragments d'un réel titubant, souvent au ras des choses : «el lápiz sobre la mesa », «el movimiento que comienza en los balcones ${ }^{50}$. Et si les recueils manifestent une exploration minutieuse du visible et du sensible, c'est souvent pour en capturer l'incertaine définition, écho direct à la langue et à l'écriture. On verra alors dans l'usage impeccable de la métrique (notamment des premiers recueils) un recours susceptible d'apaiser la désappartenance, l'appartenance précaire.

$\mathrm{Au}$ cœur de la création poétique en espagnol, un mouvement de flux et reflux, un " défaire, un refaire, battement vital $»^{51}$ tend ainsi la langue entre retournement, regard vers l'origine, tension orphique vers l'italien suspendu à la perte, et avancée trouble dans l'espagnol d'adoption, depuis la nuit. Chez Morábito, il n'y a ni point de départ ni point d'arrivée mais consistances précaires des deux idiomes. Érosion et résistance d'une part, précarité et pérennité de la langue d'adoption... Érosion et résistance de la langue maternelle dans l'ultime repli intime :

Es un hueso duro de roer. Cuando se cree que por fin nos liberamos de sus palabras, sus giros sintácticos, sus modismos intraducibles a otros idiomas, y que después de tantos años de hablar, soñar, amar e injuriar en otra lengua, uno se ha emancipado de su atadura, resulta que, al igual que esas calcificaciones de materia marina que se adhieren al cuerpo de las ballenas y que semejan enormes quistes, el viejo idioma no ha desaparecido, sólo se ha replegado en ciertas zonas, una de las cuales, quizá la más resistente, es el llanto. ${ }^{52}$

L'écriture, la nuit, forme des constellations d'images et de sons, des galeries de portraits du poète veilleur, éveilleur et voyeur, à l'écart de la présence mondaine ou dans la fascination du vis-à-vis. S'élabore dans ce séjour nocturne une poésie réflexive qui n'a de cesse de faire retour sur son advenue, sur ses conditions de possibilité et ce qu'elle transporte de la palpitation des origines. Précisément, la nuit s'étend ainsi à la langue du poème, au plus près de la matière des mots. L'espagnol tend vers son invisible, a priori indécelable dans les vers mais pourtant perpétuellement présent dans la partition sonore et la circulation métaphorique de l'ailleurs, et toujours présenté aussi par le questionnement métapoétique. La nuit, comme vecteur d'introspection et de silence fait entendre la résonance enfouie de la langue première, maternelle, aux effets/échos/bruissements acousmatiques, phénomènes vibratoires où s'abîme le poème. 
Nocturnité, labilité, profondeur, le travail poétique de Fabio Morábito révèle une langue de «l'entre-écrire ». La poésie se donne ainsi comme un autre partage des mots, entre partition verbale du lointain profond et oublieux et brèche intime, partition habitée comme une faille et invention du double du poème dans la proximité.

\section{BIBLIOGRAPHIE}

Bailly, Jean-Christophe. L'Instant et son ombre. Paris : Seuil, 2008.

Blanchot, Maurice. L'Écriture du désastre. Paris : Gallimard, 1997.

Esteban, Claude. Le Partage des mots. Paris : Gallimard, 1990.

---. Veilleurs aux confins. St-Clément : Fata Morgana, 1978.

---. L'Ordre donné à la nuit. Paris : Verdier, 2005.

Dubost, Jean-Pascal. Du travail. Strasbourg : L’Atelier contemporain, 2019.

Durand, Gilbert. Les Structures anthropologiques de l'imaginaire. Paris : Dunod, 1992 [1969].

Foessel, Michaël. La Nuit. Vivre sans témoin. Paris : Autrement, 2017.

Galinier, Jacques et Monod-Becquelin, Aurore. Las cosas de la noche, una mirada diferente. Mexico :

Edition CEMCA, 2016.

Gleize, Jean-Marie. À Noir. Poésie et littéralité. Paris : Seuil, 1992.

Guedea, Rogelio y Cortés Montes, Jair. A contraluz. Poéticas y reflexiones de la poesía mexicana reciente. México : Fondo editorial Tierra adentro, 2005.

Jabès, Edmond. Le Livre de Yukel. Paris : Gallimard, 1964.

---. Le Seuil Le Sable, Poésies complètes 1943-1988. Paris : Gallimard, « Poésie », 1990.

Meschonnic, Henri. Poétique du traduire. Paris : Verdier, 1999.

Montandon, Alain (Dir.). Dictionnaire littéraire de la nuit. Paris : Honoré Champion, 2013.

---. Promenades nocturnes. Paris : L'Harmattan, 2009.

Morábito, Fabio. Lotes baldíos. México : FCE, 1985.

---. De lunes todo el año. México : Joaquín Mortiz, 1992.

---. Alguien de lava. México : Era, 2002.

---. La ola que regresa. México : FCE, 2006.

---. También Berlín se olvida. México : Sexto Piso, 2006 [Tusquets, 2004].

---. Delante de un prado una vaca. México : Era, 2011.

---. Ventanas encendidas. Madrid : Visor de Poesía, 2012.

---. El idioma materno. México : Sexto piso, 2014.

---.« El escritor en busca de una lengua ». In Vuelta. México : 1993. 
Portante, Jean. « Le travail de la baleine ». In Pour La Poésie. Poètes de langue française (XX-XXI siècle), Paris : PUV, 2016.

Quillier, Patrick. «L'esprit coule de source ». In Continents manuscrits [En ligne], 6 | 2016, mis en ligne le 31 octobre 2016, consulté le 28 mars 2019. URL : http://journals.openedition.org/coma/ 668

---. « Pour une poétique de la vibration : acousmates, souffle, mélismes dans Trois leçons de ténèbres de José Angel Valente et Le Chant très obscur de la langue de Jacques Rebotier ». In Revue de littérature comparée, $\mathrm{n}^{\circ}$ 308. Paris : Klincksieck, 2003/4.

\section{NOTES}

1. Jabès, Edmond. Le Livre de Yukel. Paris : Gallimard, 1964, p. 245-246.

2. Nous proposons ici d'explorer les quatre recueils qui composent l'œuvre poétique ainsi que les récits et essais ouvertement méta-poétiques présentés plus loin. Toutefois, la présence de la nuit dans les nouvelles constitue à coup sûr une perspective critique à suivre.

3. On rappellera d'emblée avec Delphine Mercier que si la nuit est « conçue presqu'exclusivement comme complémentaire ou inverse du jour, [la] nocturnité implique une idée de transformation, induite par les changements qui affectent les données physiques - extérieures et internes au corps - expérimentées par les humains, et l'interprétation culturelle qu'ils en fournissent ». In “ Jacques Galinier et Aurore Monod Becquelin, Las cosas de la noche, una mirada diferente ", IdeAs [En ligne], 10 | Automne 2017 / Hiver 2018, mis en ligne le 18 décembre 2017, consulté le 19 avril 2019 (consultable sur http://journals.openedition.org/ideas/2175). Comme nous l'avons indiqué dans notre texte d'introduction à ce volume, le terme constitue en effet un des axes clés du groupe de recherche " Anthropologie de la nuit " (Laboratoire d'ethnologie et de sociologie comparative / CNRS-Université Paris Ouest) qui le définit ainsi : «Le concept de "nocturnité » rassemble les éléments qu'une société formule et adopte pour définir ce qui constitue «sa" nuit, conjointement au temps physique et en même temps au delà de cette période qui s'étend du lever au coucher héliaque. Les espaces, les temps et les notions qui définissent la nocturnité dans une société donnée s'incarnent en un certain nombre de personnages, d'objets, de ressentis, de modification des affects, de comportements, de discours, qui se manifestent particulièrement à certaines occasions rituelles, soit « de nuit » soit " hors la nuit », et peuvent même s'incruster durablement dans le temps diurne » (consultable sur http://lesc-cnrs.fr/fr/anthropologie-de-lanuit), et fait aussi l'objet d'un usage critique chez nombre de chercheurs sur les Ressources Environnementales Nocturnes, tOurisme, territoIRes (renoir), S. Challéat notamment.

4. Morábito, Fabio. El idioma materno. México : Sexto Piso, 2014, p. 137. En correspondance avec un lecteur hibou : «siendo la lectura una actividad fundamentalmente nocturna, aunque se haga de día. El que lee, abandona la realidad por la escritura; el penetrar en el recinto sellado de lo escrito lo vuelve ciego frente al mundo, en su alma se hace de noche y él se convierte en otra criatura... » 5. Blanchot, Maurice. L'Écriture du désastre [1980]. Paris : Gallimard, 1997, p. 185. On pensera également à la définition de l'acte d'écrire proposée par Michaël Foessel comme expérience d'essence nocturne : «qui montre les choses sous un nouveau jour [...], qui réclame un certain degré d'obscurité », La Nuit. Vivre sans témoins. Paris : Autrement, 2017, p. 15.

6. Morábito, Fabio. Lotes baldíos. México : FCE, 1985; De lunes todo el año. México : Joaquín Mortiz, 1992 ; Alguien de lava. México : ERA, 2002 ; Delante de un prado una vaca. México : Era, 2011.

7. Reprenant le beau titre de Claude Esteban, Veilleurs aux confins. St-Clément : Fata Morgana, 1978.

8. Morábito, Fabio. El idioma materno. Op. cit., p. 61. 
9. Déclinant le néologisme poétique « effaçonner/effaçonnement » de Jean Portante sur lequel nous reviendrons.

10. Morábito, Fabio. También Berlín se olvida. México : Sexto piso, 2006, p. 71.

11. Foessel, Michaël. Op. cit., p. 8.

12. Montandon, Alain. Promenades nocturnes. Paris : L'Harmattan, 2009, p. 8.

13. Dubost, Jean-Pascal. Du travail. Strasbourg : Éditions L'Atelier contemporain, 2019, p. 20.

14. Morábito, Fabio. El Idioma materno. Op. cit., p. 86 et 146 pour les deux fragments cités.

15. Patrick Quillier, citant Giorgio Agamben sur la poésie de Valente, «Pour une poétique de la vibration: acousmates, souffle, mélismes dans Trois leçons de ténèbres de José Angel Valente et Le Chant très obscur de la langue de Jacques Rebotier ", in Revue de littérature comparée, $n^{\circ} 308$. Paris, Klincksieck, 2003/4, p. 503.

16. Morábito, Fabio. El idioma materno, Op. cit., p. 105.

17. Morábito, Fabio. «In limine », Lotes baldíos. Op. cit., p. 13-14.

18. Morábito, Fabio. «Tres ciudades 1. Último de la tribu », idem, p. 17.

19. "Tres ciudades 2. Milán », idem, p. 22.

20. «Tres ciudades 3. Ciudad de México », ibid, p. 23.

21. Une contention exemplairement exprimée ici dans la forme de l'heptasyllabe, et par la sobriété du style à l'échelle de l'œuvre. On ne sera pas surpris, aussi, de découvrir avec le voyageur Morábito une ode à la concision, rendue avec humour dans le bref fragment suivant : "Ahora podría decir que siempre he escrito poesía como quien comprime lo esencial de sus pertenencias en una valija de poco peso, porque se marcha a un lugar que no conoce y no quiere cargar un bulto voluminoso, y me temo que tampoco esta vez se me tomaría en serio si afirmara que mi mayor influencia literaria no es tal o cual poeta insigne, sino la línea de maletas Samsonite", El idioma materno. Op. cit., p. 90.

22. Meschonnic, Henri. Poétique du traduire. Paris : Verdier, 1999, p. 459.

23. Morábito, Fabio. "Ventanas encendidas ». In Ventanas encendidas. Antología poética. Madrid: Visor, 2012, p. 139.

24. La première, La ola que regresa (México : FCE, 2006), réunissait les trois premiers recueils alors publiés.

25. Bailly, Jean-Christophe. L'Instant et son ombre. Paris : Seuil, 2008, p. 26.

26. On dira ainsi la qualité optique et symbolique du poème comme fenêtre, comme trappe, conformément à l'usage du terme, entre autres, par Raphaël Célis : « cette fenestrité, essentielle à l'imaginaire, par quoi se pratique l'ouverture et l'échange entre le monde de l'apparence et le monde réel », p. 64, in La mondanité du jeu et de l'image selon Eugen Fink. Revue Philosophique de Louvain. Quatrième série, tome $76, \mathrm{n}^{\circ} 29,1978$. pp. 54-66.

27. Morábito, Fabio. « Verso y prosa ». In El idioma materno. Op. cit., p. 51-52.

28. Morábito, Fabio. El idioma materno. Op. cit., «Robar »p. 14 et «Ladrón y centinella », p. 15-16 pour les deux fragments cités.

29. On songera au couple «brigand/poète » évoqué par Louis-Sébastien Mercier (L'Incertitude du regard, Paris, La Découverte Poche, 2006) glosé par Michaël Foessel. Op. cit., p. 19.

30. L'expression revient à Jean-Michel Maulpoix, « Extraire la beauté du trivial », une conférence organisée par le groupe de recherche 'Cultures Anglo-Saxonnes' (CAS) dans le cadre de l'axe de recherche Poétiques, et le groupe de recherche 'Patrimoine, Littérature, Histoire' (PLH), Université de Toulouse-Le Mirail (UT2J), 4 décembre 2012.

31. Morábito, Fabio. El idioma materno. Op. cit., p. 72.

32. Maulpoix, Jean-Michel. La Poésie a mauvais genre. Paris : Ed. José Corti, 2016.

33. Jabès, Edmond. «Terres d'outre-nuit que le soleil arrache à la méditation et aux épines du doute ». In Le Seuil Le Sable, Poésies complètes 1943-1988. Paris : Gallimard, « Poésie », 1990, p. 27.

34. Morábito, Fabio. Delante de un prado una vaca. Op. cit., p. 107-108. 
35. Portante, Jean. «Le travail de la baleine». In https://www.cairn.info/pour-lapoesie--9782842924560-page-147.htm

36. Morábito, Fabio. El idioma materno. Op. cit., p. 71 et 122 pour les deux références.

37. « La escritura [...] ha inventado el silencio y la inmersión en profundidad », El idioma materno. Op. cit., p. 136.

38. Morábito, Fabio. « Mi padre siempre trabajó en lo mismo ». In Alguien de lava. Op. cit., p. 30.

39. Morábito, Fabio. El idioma materno. Op. cit., p. 106.

40. Gilbert Durand, dans une glose de Bachelard citant Lawrence : «l'oreille peut entendre plus profondément que les yeux ne peuvent voir ", Les Structures anthropologiques de l'imaginaire. Paris : Dunod, 1992 [1969], p. 99.

41. Quillier, Patrick. «Pour une poétique de la vibration: acousmates, souffle, mélisses dans Trois leçons de ténèbres de José Angel Valente et Le Chant très obscur de la langue de Jacques Rebotier. In Revue de littérature comparée, $\mathrm{n}^{\circ}$ 308. Paris : Klincksieck, 2003/4, p. 494.

42. Morábito, Fabio. «El escritor en busca de una lengua ». In Vuelta. México : Vuelta, 1993, p. 21.

43. L'auteur rend d'ailleurs hommage au poète péruvien en s'en revendiquant veilleur : «El velador de Vallejo ", El idioma materno. Op. cit., p. 41-42.

44. Esteban, Claude. Le Partage des mots. Paris : Gallimard, 1990, p. 23.

45. Morábito, Fabio. «El escritor en busca de una lengua », Art. cit., p. 20.

46. Morábito, Fabio. «A Mariapía Lamberti ». In De lunes todo el año. Op. cit., p. 63.

47. Morábito, Fabio. « El escritor en buca de la lengua ». Art. cit., p. 21.

48. Morábito, Fabio. Idem.

49. Esteban, Claude. Idem.

50. Morábito, Fabio. Ventanas encendidas. Op. cit., p. 107.

51. Quillier, Patrick. «L'esprit coule de source ». In Continents manuscrits [En ligne], 6 | 2016, mis en ligne le 31 octobre 2016, consulté le 28 mars 2019. URL : http://journals.openedition.org/ coma/668.

52. Morábito, Fabio. El idioma materno. Op. cit., p. 177.

\section{RÉSUMÉS}

Dans le séjour de la nuit où palpitent les origines (Alexandrie, Milan, Mexico), opère chez l'auteur italo-mexicain Fabio Morábito (Alexandrie, 1955) une mise en marche de la langue vers l'inflammation poétique. Depuis le tout premier recueil, l'écriture se lie intimement à la nuit où elle se loge, se construit et se pense dans l'entre-deux langues, écriture aux confins du premier idiome - l'italien familial et nomade -, et d'un espagnol malgré lui palimpseste du souffle intime. Aussi, le geste lyrique semble interroger toujours, dans l'élan des vers, la part de l'avancée et celle du retournement d'une voix sans cesse reconquise à l'étrangeté.

On interrogera ici le désir de nuit d'un poète veilleur aux confins, à la fois veilleur et éveilleur de la sensibilité nocturne, éveilleur de la langue en contre-jour du poème et voyeur d'avant le jour, poète vagabond, voleur furtif de mots et d'images ou vampire iconique. Il sera aussi un " effaçonneur ", faisant de la nuit de la langue - la nocturnité de l'italien laissé derrière -, la respiration profonde, la rythmique secrète et perturbante de poèmes d'outre-nuit.

In the night's stay, where pulsate the origins (Alexandria, Milan, Mexico), Italo-Mexican poet Fabio Morábito (Alexandria, 1955) works to turn the language towards poetic inflammation. 
Since the very first collection, writing intimately binds to the night, where it is lodged, built and thought of in an inbetweenness, writing on the borders of the first idiom - the family and nomadic Italian - and of the adopted Spanish as the palimpsest of the intimate breath. So the lyrical gesture always seems to question, in the momentum of the verses, the part of the advance and that of the reversal of a voice constantly reconquered to strangeness.

We intend here to examine the desire of night of a poet both "watchman" on the edge and arouser of the nocturnal sensibility, awakener of the backlight language of the poem and nocturnal voyeur, poet wanderer, stealthy stealer of words and images or iconic vampire. He will be also a "shaper", transforming the night of the language - the nocturnality of the Italian left behind - into deep breathing, into secret and disturbing rhythm of poems captured through dark.

\section{INDEX}

Keywords : Fabio Morábito, night writing, nocturnal language, bilingualism, acousmatic, inbetweenness

Mots-clés : Fabio Morábito, écriture nocturne, nocturnité de la langue, bilinguisme, acousmatique, entre-deux

\section{AUTEURS}

\section{NATHALIE GALLAND}

Maître de conférences

Université de Bourgogne Franche-Comté

nathalieboudon@hotmail.com 